\title{
MODULAR RECONSTRUCTION SYSTEMS - FINAL SOLUTION FOR CRITICAL BONE DEFECTS TREATMENT
}

doi: $10.2478 /$ rojost-2018-0024

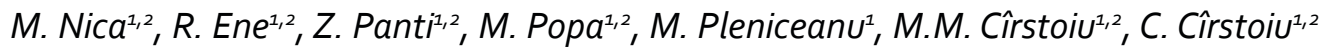 \\ ${ }^{1}$ University Emergency Hospital, Bucharest, Romania \\ 2"Carol Davila" University of Medicine and Pharmacy, Bucharest, Romania
}

Introduction. The management of critical bone defects has experienced an unprecedented improvement in the last few decades. A good primary mechanical stability and versatility of the modular reconstruction systems allowed the conversion to universal setups and the expansion of indication. The progression and reliability of the reconstruction possibilities available nowadays are outlined using the experience of our department.

Material and method. A retrospective review of critical bone defect cases and the reconstruction management using endoprosthetic systems is presented. Ten reconstruction surgeries performed in the last 18 months in our department were observed. The patients were 3 males and 7 females with a mean age of 45.3 (range 19 to 75 ). Modular systems were used for the reconstruction of bone defects subsequent to resections of benign or malignant musculoskeletal tumors (76\%) and to non-neoplastic conditions (24\%).

Results. On the latest follow-up, all the patients were alive, with one case of tumoral recurrence. No aseptic loosening, soft tissue or structural failure were recorded following surgery, with one case under treatment for infection after it required an open procedure for a prosthetic hip dislocation. The early functional results were adequate in all cases, with good secondary stability and satisfactory quality of life.

Conclusion. The evolution of endoprosthetic reconstruction surgery has improved the prognosis for many patients diagnosed with critical bone defects.

Continuous advances in implant design, fixation, and soft tissue attachment combined with the improvement of surgical techniques will ensure a bright future for this type of procedures.

Keywords: reconstruction, bone defect, modular system 